\title{
MEDIDA DA PRESSÃO ARTERIAL EM GESTANTE
}

Sonia M. Junqueira V. de Oliveira*

Edna Apparecida Moura Arcuri**

OLIVEIRA, S.M.J.V.; ARCURI, E.A.M. Medida da pressão arterial em gestante. Rev.latino-am.enfermagem, Ribeirão Preto, v. 5, n. 3, p. 49-55, julho 1997.

Trata-se de revisão da literatura à respeito da medida indireta da pressão arterial (P A) em gestante normotensa. Aborda as modificações ocorridas na pressão arterial sistólica e diastólica decorrentes da gravidez. São discutidos aspectos polêmicos no procedimento de medida da P A, como por exemplo qual a fase dos sons de Korotkoff (fase quatro ou cinco) que representa melhor a pressão diastólica e o uso da Monitorização Ambulatorial da Pressão Arterial. Enfatiza as recomendações de diferentes sociedades (American Heart Association, British Hypertension Society, Australasian Society, National High Blood Pressure Education Program e World Health Organization).

UNITERMOS: pressão arterial, medida de pressão em gestante

\section{INTRODUÇÃO}

A finalidade precípua desta publicação é apresentar ao leitor uma revisão da literatura referente a medida da pressão arterial em gestante.

A doença hipertensiva específica da gravidez constitue uma das mais importantes complicações do ciclo gravídico-puerperal, entre outras patologias que podem estar manifestadas ou agravadas durante este período, apresentando alto risco de morbimortalidade para o binômio mãe-filho. A incidência da DHEG é muito variável entre os diferentes países e mesmo entre as regiões de um mesmo país, a julgar pelo estudo internacional conduzido pela WORLD HEALTH ORGANIZATION (1988), que refere incidência de hipertensão na gravidez de $33 \%$ na China, 26\% na Tailândia, 7\% em Burma e 5\% no Vietnã, enquanto BARRON \& LINDHEIMER (1995) e NEME (1994) apontam que a incidência desta moléstia está em torno de $10 \%$. Considerando que a DHEG, ainda nos dias de hoje, próximo ao final do século, não possa ser prevenida na sua forma de pré-eclâmpsia, sua gravidade maior, eclâmpsia pode, pela assistência pré-natal mais assídua e rigorosa. Nesse sentido os níveis da pressão arterial é o parâmetro mais importante para o diagnóstico da doença hipertensiva específica da gravidez. Assim, a medida precisa da pressão arterial é uma condição imprescindível na avaliação clínica da gestante, durante o pré-natal, com o objetivo de diagnosticar precocemente a DHEG, uma vez que o aumento da pressão arterial é, geralmente, o primeiro sinal clinico da doença (NATIONAL High Blood Pressure Education Program, 1990).

\section{MUDANÇAS FISIOLÓGICAS RELACIONADAS Á PRESSÃO ARTERIAL}

Durante a gravidez ocorrem modificações no sistema cardiovascular materno, sendo que o aumento da volemia determina o aumento do débito cardíaco (RUDGE \& BEREZOWSKI, 1994), incremento este que pode atingir 30 a $40 \%$ do débito normal, como informa o NACIONAL High Blood Pressure Education Program (1990). O aumento do débito cardíaco deveria resultar em aumento da PA. Entretanto, este fato não ocorre devido à diminuição acentuada da resistência vascular periférica, que é decorrente de alterações bioquímicas nos vasos maternos induzidas pelo sistema das prostaglandinas (RUDGE \& BEREZOWSKI, 1994). Segundo WALLENBURG (1988), existe aumento na produção de prostaciclina (PGI 2) em relação ao tromboxane (TXA 2), promovendo uma vasodilatação generalizada com consequente diminuição da resistência vascular.

MARTINS (1982) chama a atenção para a influência do mecanismo fistuloso ao nível da placenta, que explica a queda da pressão arterial diastólica,

\footnotetext{
* Professor Assistente do Departamento de Enfermagem Materno-Infantil e Psiquiátrica da Escola de Enfermagem da USP

** Professor Titular do Departamento de Enfermagem Médico-Cirúrgica da Escola de Enfermagem da USP
} 
principalmente durante o $2^{\circ}$ trimentre da gestação. Complementando, WILSON et al. (1980) referem que o aumento da atividade das protaglandinas ou o "Shunt" arterio-venoso causado pela unidade útero-placenta pode provocar diminuição na resistência vascular periférica, com conseqüente vasodilatação que poderia expandir a capacidade intravascular, levando à diminuição na PA.

O interesse pela evolução da pressão arterial durante a gravidez é bastante antigo, desde que Schedoff e Porockjakoff, em 1884, chamaram a atenção para a associação do aumento da pressão arterial com a eclâmpsia (HENRY, 1936). Desde então, muitos estudos têm sido publicados à respeito das modificações na PA em decorrência da gestação, dentre eles podemos citar os conduzidos por HARE \& KARN (1929); HENRY (1936); ANDROS \& MICH (1945); MACGILLIVRAY et al. (1969); WILSON et al. (1980); MARGULIES et al. (1987); REISS et al. (1987); VOLDMAN et al. (1988); BEREZOWSKI et al. (1989) e VILLAR et al. (1989). Tais estudos tiveram por objetivos conhecer o valor da pressão arterial normal com a finalidade de reconhecer os desvios patológicos que caracterizam a doença hipertensiva específica da gravidez.

A primeira publicação sobre os padrões normais da pressão arterial durante a gestação foi de HARE \& KARN (1929). Estudando a pressão arterial, o pulso e a resposta ao exercício durante a gravidez normal, estes autores verificaram que a pressão arterial sistólica (PAS) durante a gestação era menor, quando comparada com mulheres não grávidas, apresentando uma tendência a valores mais baixos entre a $21^{\mathrm{a}}$ e $28^{\mathrm{a}}$ semanas e a partir daí evidenciaram um leve aumento. Quanto à pressão arterial diastólica (PAD), os autores não constataram diferença significativa em relação aos níveis de pacientes não grávidas. Dados controversos foram obtidos por HENRY (1936), que pesquisando os efeitos da gestação sobre a pressão arterial observou níveis mais baixos na gestante do que na mulher fora da gravidez, principalmente para os níveis da PAD.

Comparando os níveis da PA pré-gravídicos e durante a gestação normal de uma mesma mulher, ANDROS \& MICH (1945) também evidenciaram valores levemente mais baixos para a PAD no primeiro e segundo trimestres, sendo que no terceiro trimestre a PAD apresentou valores semelhantes aos níveis pré-gravídicos. Neste estudo os autores não constataram modificação na PAS durante a evolução da gravidez.

Preocupados com o erro do observador, evidenciado na década de 60, MACGILLIVRAY et al. (1969) desenvolveram em Londres um dos estudos prospectivos mais citados na literatura, utilizando o esfigmomanômetro random -zero. Analisaram a evolução da PA em 226 primigestas, durante a gravidez até a $6^{\text {a }}$ semana após o parto. Encontraram valores mais baixos tanto para a PAS como para a PAD, no intervalo da $16^{\mathrm{a}}$ à $20^{\mathrm{a}}$ semana da gestação, sendo que posteriormente ocorreu um aumento progressivo com o avanço da gravidez, aumento este substancialmente menor na PAS do que na PAD.

WILSON et al. (1980) também analisaram a modificação da PA e do sistema renina-aldosterona em 69 mulheres durante a gravidez até a $6^{\mathrm{a}}$ semana após o parto. Concluíram que os valores da média da PA foram mais baixos durante a gestação do que os valores encontrados no puerpério, com maior declínio da PAD comparado a PAS. Os valores mais baixos da PAD ocorreram próximo da $28^{\mathrm{a}}$ semana e a partir daí tenderam a retornar aos níveis pré-gravídicos. Resultados controversos foram encontrados por MARGULIES et al. (1987), numa população de 249 gestantes normais da América-Latina (Argentina e Uruguai), onde a PAS e a PAD permaneceram constantes no intervalo de 10 a 34 semanas da gravidez, sendo que ao final da gestação houve um aumento de aproximadamente $10 \mathrm{mmHg}$ para a PAS e $5 \mathrm{mmHg}$ para a PAD.

Em outro estudo utilizando o esfigmomanômetro random-zero, VILLAR et al. (1989), encontraram para a PAS e PAD valores aumentados após a $30^{\mathrm{a}}$ semana de gestação, atingindo o ponto mais alto no termo. A média dos valores da PAS com 20 semanas de gestação foi de $102 \mathrm{mmHg}$ e com 41 semanas de $112 \mathrm{mmHg}$, enquanto a PAD foi de $62 \mathrm{mmHg}$ e $72 \mathrm{mmHg}$, respectivamente. Em contrapartida, o estudo de REISS et al. (1987) mostrou uma queda da PA antes da metade da gravidez (como sugerem os autores anteriores), ou seja, por volta da $9^{\mathrm{a}}$ à $12^{\mathrm{a}}$ semana, sendo que estes níveis permaneceram baixos durante toda a gestação. Vale a pena ressaltar, no entanto, que neste estudo a posição utilizada para medir a PA foi o decúbito lateral esquerdo, que conforme demonstrado por MACGILLIVRAY et al. (1969), tem induzido a níveis mais baixos de PA, quando comparado com a posição sentada .

Em estudo nacional realizado no Brasil por BEREZOWSKI et al. (1989), os valores da PAS e PAD durante a gravidez normal permaneceram em níveis baixos entre a sétima semana e o segundo trimestre, tendendo a retornar a níveis mais elevados a partir da $32^{\mathrm{a}}$ a $34^{\text {a }}$ semanas.

Considerando as diferentes posições (em pé, decúbito dorsal horizontal e decúbito lateral esquerdo) WICHMAN \& RYDÉN (1986) verificaram que a PAD sofreu um aumento de $15 \mathrm{mmHg}$ ou mais o início do $2^{\circ}$ trimestre até a $36^{\mathrm{a}}$. semana de gravidez, em $25 \%$ dos casos estudados.

Analisando 1000 casos de primigestas normais na Venezuela VOLDMAN et al. (1988) perceberam que a PAS apresentou um aumento discreto no último trimestre e a PAD aumentou desde o primeiro até o terceiro trimestre. 
Com o objetivo de determinar o padrão da PA na gestante nigeriana, OKONOFUA et al. (1992) fizeram um estudo longitudinal em 189 grávidas, no qual evidenciaram que a PAS, PAD e pressão arterial média (PAM) mostravam uma queda na metade da gestação. A média de PA mais baixa foi registrada entre a $24^{\mathrm{a}}$ e $26^{\mathrm{a}}$ semanas da gravidez; posteriormente, todas as medidas de pressão aumentaram progressivamente até o termo.

Percebe-se que os pesquisadores de diferentes locais estabelecem valores considerados "normais" para a PA durante a gravidez, numa determinada população; nesse sentido, MACGILLIVRAY et al. (1969) chamam atenção para a existência de valores diferentes de PA quando se considera as diferenças étnicas.

O Ministério da Saúde, por sua vez, afirma que sendo uma variável contínua a pressão arterial tem distribuição e médias diferentes, dependendo da população selecionada e, portanto, os valores considerados normais derivados de uma amostra, podem ter significado clínico inteiramente distinto, quando aplicados a outra população (BRASIL, 1993).

\section{MONITORIZAÇÃO AMBULATORIAL DA PRESSÃO ARTERIAL}

Além dos aspectos anteriormente citados sabese que a pressão arterial é uma variável hemodinâmica, que sofre variação durante o dia todo, guardando relação com o ritmo circadiano. Devido à impossibilidade de fornecer a variação circadiana da pressão arterial pela esfigmomanometria de Riva Rocci, no final da década de 70 foi introduzido o primeiro sistema com monitor portátil automático e não invasivo, que permitia o registro dos níveis da pressão em diversos momentos das 24 horas, independentemente do local onde o cliente se encontrasse (MANCIA \& ZANCHETTI, 1985 e AMODEO, 1995). Tal sistema é conhecido no Brasil como monitorização ambulatorial da pressão arterial (MAPA).

As vantagens de poder avaliar a pressão arterial da gestante normotensa ou hipertensa nas 24 horas do dia tem suscitado muitas pesquisas (CLARK et al., 1991; RAYBURN \& ZUSPAN, 1992; BROWN et al., 1993 e OLOFSSON \& PERSON, 1995). Segundo LUDERS et al. (1995), a MAPA é um método satisfatório na medida da PA, uma vez que se baseia em grande número de medidas e elimina a variabilidade de leitura entre diferentes observadores, além de reduzir erros pela ocorrência de hipertensão do "avental branco". Comparando os valores da pressão arterial obtidos através da MAPA e com esfigmomanômetro random-zero, BROWN et al. (1993) evidenciaram que estando a gestante sentada, a MAPA hiperestima a pressão sistólica em $5 \mathrm{mmHg}(\mathrm{p}<0,0001)$ e hipoestima a pressão diastólica fase $4 \mathrm{em} 7 \mathrm{mmHg}(\mathrm{p}<0,001)$ e a fase $5 \mathrm{em} 3 \mathrm{mmHg}$ $(\mathrm{p}<0,01)$.

Resultados semelhantes são descritos por OLOFSSON \& PERSON (1995), que ao comparar em 99 gestantes a MAPA de 24 horas com a medida casual da PA, realizada com esfigmomanômetro de mercúrio, constataram que a média da pressão sistólica verificada pela MAPA foi $4,6 \mathrm{mmHg}$ mais elevada $(\mathrm{P}<0,001)$, enquanto a média da pressão diastólica foi $2,7 \mathrm{mmHg}$ mais baixa $(\mathrm{P}<0,001)$, em relação ao método tradicional de medida da pressão. Concluiram os autores que, apesar das diferenças serem pequenas em termos absolutos de valores em $\mathrm{mmHg}$, a monitoragem da pressão pode resultar em altas taxas de falso positivo (35\%) e falso negativo (20\%) no diagnóstico da hipertensão, e que a MAPA de 24 horas não pode substituir o método tradicional de medida da pressão, a não ser que novas definições de hipertensão sejam estabelecidas.

Utilizando a MAPA de 24 horas na gestação, HALLIGAN et al. (1993) fizeram um estudo prospectivo em 98 primigestas caucasianas, durante o primeiro, segundo e terceiro trimestres da gravidez, e também 6 semanas após o parto. Constataram aumento significante $(\mathrm{p}<0,001)$ da pressão sistólica, durante o período diurno e noturno, a partir da $33^{\mathrm{a}}$ até a $40^{\mathrm{a}}$ semana em relação aos valores do primeiro trimestre; na pressão diastólica observaram queda entre a $18^{\mathrm{a}}$ e $24^{\mathrm{a}}$ semana nos dois períodos e aumento a partir da $25^{\mathrm{a}}$ semana, até o fim da gravidez. Verificaram também que a pressão diastólica 6 semanas após o parto $(76 \mathrm{mmHg})$ apresentava-se significativamente maior $(\mathrm{p}<0,001)$, que a registrada no primeiro trimestre $(70 \mathrm{mmHg})$. A queda noturna da $\mathrm{PA}$ foi preservada durante toda a gravidez. A medida casual da pressão arterial pelo método auscultatório comparada com a média do dia da MAPA, revelou-se significativamente mais elevada tanto para a pressão sistólica $(8 \mathrm{mmHg})$ como para a diastólica $(9 \mathrm{mmHg})$, até a $33^{\mathrm{a}}$ semana; convém ressaltar que foi utilizada a fase 4 para a medida da pressão diastólica pelo método tradicional, enquanto a MAPA utiliza a fase 5 .

Tomando-se por base os parâmetros do NATIONAL High Blood Pressure Education Program (1990) ocorre uma diminuição média de 7 a 10mmHg na PAD, no início da gestação normal, com aumento no terceiro trimestre, atingindo então os níveis prégravídicos; quanto à PAS parecem existir apenas pequenas modificações.

\section{DETERMINAÇÃO DA PRESSÃO ARTERIAL DIASTÓLICA: FASE 4 OU 5?}

Na medida da pressão arterial um aspecto 
bastante controverso é a respeito de qual fase dos sons de Korotkoff - fase 4 (abafamento do som) ou fase 5 (desaparecimento do som) representa com mais precisão os níveis intra-arteriais da PAD durante a gestação. Este fato tem gerado recomendações divergentes sendo difundido o uso de ambas as fases. De acordo com as recomendações do NATIONAL High Blood Pressure Education Program (1990) a PAD na gestante é definida como o início da fase 5, pois esta fase correlaciona melhor com a pressão intra-arterial. Entretanto, este grupo recomenda que em algumas gestantes que não apresentam a fase 5 distinguível (isto é, o som de Korotkoff não desaparece e pode ser auscultado até próximo de zero $\mathrm{mmHg}$ ), a fase 4 deve também ser registrada, apesar da dificuldade em identificar o abafamento, e assim ambas as fases 4 e 5 devem ser anotadas. Esta recomendação também foi citada na última publicação da American Heart Association (PERLOFF et al., 1993).

Posição diferente foi adotada pela British Hypertension Society (PETRIE et al., 1986), pela WORLD HEALTH ORGANIZATION (1987) e AUSTRALASIAN SOCIETY FOR THE STUDY OF HYPERTENSION IN PREGNANCY (1993) que recomendam o uso da fase 4 de Korotkoff para todas as gestantes. Por outro lado, pesquisas recentes tem mostrado uma grande diversidade na forma de registro da pressão diastólica. BISSON et al. (1991) encontraram $42 \%$ dos médicos e enfermeiras obstétras ingleses utilizando a fase 5 (desaparecimento do som). Resultado semelhante foi encontrado por PERRY et al. (1991 a), em estudo sobre a prática atual da equipe obstétrica em verificar a pressão arterial de grávidas, constatando que $53 \%$ deles utilizam a fase 5 de Korotkoff na determinação da pressão diastólica, apesar da recomendação contrária da British Hypertension Society.

Esta questão polêmica pode ser resolvida comparando-se a pressão intra-arterial com aquela verificada de forma indireta. Comparando a medida direta e indireta da pressão arterial, utilizando um esfigmomanômetro random-zero, em 50 gestantes, RAFTERY \& WARD (1968) encontraram valores da medida indireta hiperestimando a pressão arterial diastólica em $11 \mathrm{mmHg}$ na fase 4 e $7 \mathrm{mmHg}$ na fase 5 , sendo que em $72 \%$ da população a fase 5 foi a que mais se aproximou dos valores intra-arteriais. Resultados diferentes foram relatados por MILSON et al. (1986), que observaram médias de pressão intra-arterial 4 a $7 \mathrm{mmHg}$ maior do que a fase 4 verificada com esfigmomanômetro padrão ou mesmo por microcomputador automático (Dinamap). Apesar dos valores controversos dos estudos citados, a maior parte das publicações tendem a referir uma pressão diferencial (pressão de pulso) maior na medida direta, que é geralmente mais alta na PAS e mais baixa na PAD. É fácil compreender tais diferenças do ponto de vista hemodinâmico, pois enquanto o catéter na artéria registra as oscilações da parede do vaso pela passagem do fluxo sanguíneo em alta velocidade, o método auscultatório depende de grande turbulência do fluxo, com formação de vórtices que geram os sons a serem captados pelo estetocópio. Assim, na PAS as oscilações captadas pelo método direto são iniciadas antes da geração dos sons do método indireto. $\mathrm{O}$ silêncio que indica a PAD no método indireto, ocorre antes do término das oscilações que a indicam no método direto.

\section{CONFIABILIDADE NA IDENTIFICAÇÃO DA FASE 4 OU 5}

Outro aspecto polêmico é quanto a precisão em identificar o abafamento (fase 4) e o desaparecimento do som (fase 5), uma vez que a fase 5 pode estar muito próxima ou coincidente com zero, em algumas gestantes, devido as modificações circulatórias que ocorrem no período gestacional (JOHENNING \& BARRON, 1992).

Em um dos estudos seriados mais amplos encontrados na literatura, MACGILLIVRAY et al. (1969) constataram que a fase 5 (desaparecimento do som) tinha valores próximos de zero em muitas grávidas; todavia, os dados confirmando esta afirmação não foram apresentados. Entretanto, até recentemente, poucas pesquisas existiam para avaliar a frequiência ou ausência da fase 5 na gestante.

O relatório da WORLD HEALTH ORGANIZATION (1987) refere que em aproximadamente $15 \%$ das grávidas a fase 5 está ausente, pois os sons de Korotkoff não desaparecerem e podem ser auscultados até a pressão do manômetro cair próximo de zero mmHg. Na década de 80, WICHMAN \& RYDÉN (1986) encontraram a fase 5 ausente, em $7 \%$ das medidas da PA, contudo o número de grávidas não foi mencionado. Mais recentemente, PERRY et al. (1990 e 1991a, b) e BROWN \& WHITWORTH (1991) analisando a pressão arterial de 197 e 50 gestantes, respectivamente, não encontraram valores próximos de zero para a fase 5 . Concordando com estes resultados, ao estudar 1194 gestantes, em diferentes idades gestacionais e nas posições lateral, supina e sentada, LOPES et al. (1994) observaram que a prevalência de valores zero para a fase 5 foi sempre menor do que $0,5 \%$, sendo que a menor incidência de valor zero, quando existiu, foi encontrada na posição sentada.

Quanto à detecção da $4^{\mathrm{a}}$ fase, JOHENNING \& BARRON (1992) sumarizam resultados de vários estudos que apontam uma variação de 10,2\% a 78,8\%. Percebese portanto uma considerável discrepância entre estas 
pesquisas a respeito de quantas vezes a fase 4 (abafamento do som) pode ser detectada na gestante.

Na prática, a ausculta da $4^{a}$ fase não é fácil de ser identificada, devido ser uma fase em que fisiologicamente, ocorre pequena mudança dos sons de Korotkoff, o que resulta em grande diferenciação entre os observadores e, consequentemente, em grande discrepância entre eles e nas condutas que definem a PAD

PERRY et al. (1991 b) concluem que a identificação da $4^{a}$ fase está mais sujeita a variabilidade do observador do que a $5^{\mathrm{a}}$ fase, fundamentados nas observações realizadas em gestantes e mulheres não grávidas.

Concluindo, embora alguns autores afirmem que a fase 5 é auscultada em valores muito baixo, ou mesmo não acontece absolutamente em um número significante de gestantes, existindo poucos dados embasando esta visão. Fundamentados em várias pesquisas JOHENNING \& BARRON (1992), referem que a fase 5 pode ser detectada em mais de $90 \%$ das grávidas.

Uma outra questão discutível é a diferença existente entre os valores da fase 4 e 5 . Este fato é de fundamental importância, pois os valores da fase 4 são significativamente maiores do que os da fase 5 (JOHENNIG \& BARRON, 1992). Ao utilizar-se a fase 4 para determinar a PAD, pode um número maior de gestantes ser erroneamente categorizadas e tratadas como hipertensas.

Pesquisas comparando as diferenças entre os valores da fase 4 e 5 durante a gravidez referem médias de $4 \mathrm{mmHg}$ (BROWN et al., 1994), 4,5mmHg (RAFTERY \& WARD, 1968), 5mmHg (BROWN \& WHITWORTH, 1991), 6mmHg (LOPES et al., 1994) $6,2 \mathrm{mmHg}$ (CLARK et al., 1991) e 12,5mmHg (VILLAR et al., 1989).

As controvérsias relacionadas a decisão de se utilizar a fase 4 ou 5 em gestantes resultam das mesmas dúvidas encontradas em outros grupos populacionais, como em crianças, e mesmo adultos com patologias causando grande distinção entre as duas fases, como é o caso de Insuficiência Aórtica e outras discinesias. Tais dúvidas geraram a sugestão da última publicação da American Heart Association PERLOFF et al. (1993), de se registrar as duas fases quando os ruídos da fase 5 chegam a zero, para todos os grupos populacionais. $\mathrm{Na}$ prática, parece que o registro da medida da pressão arterial na gestante deva ser claro e preciso, devendo portanto ser registrada a fase 4 , quando a fase 5 indica valores próximos de zero.

\section{CONCLUSÃO}

Publicação recente de ZANCHETTI \& MANCIA (1996) homenageia Riva Rocci, que há um século brindava a humanidade com o primeiro esfigmomanômetro construído com o princípio dos aparelhos atuais. Apesar do aperfeiçoamento dos instrumentos em decorrência ao desenvolvimento tecnológico e científico e das centenas de estudos realizados no campo da esfigmomanometria durante o século, a precisão na medida da pressão arterial ainda é um grande desafio para o século XXI. Na gestante este desafio é ainda maior, pois as modificações anátomofisiológicas que interferem na PA são muitas, sobretudo as hemodinâmicas e hormonais, tornando a sua avaliação ainda mais difícil.

Apesar das controvérsias identificadas na análise da literatura a necessidade de atualização é inquestionável, tendo em vista o perfil de morbimortalidade da mulher por Doença Hipertensiva Específica de Gravidez, revelando índices altamente preocupantes. Conforme o exposto nesta revisão, a medida de pressão arterial pelo método tradicional continua sendo de fundamental importância no diagnóstico precoce da DHEG, porém até agora pequena atenção tem sido dada a esta atividade no cuidado prénatal, na maioria dos ambientes obstétricos (VILLAR et al. 1989).

\section{BLOOD PRESSURE MEASUREMENT IN PREGNANCY}

This study deals with the review of the literature regarding the indirect blood pressure measurement in normal pregnant women. It shows the changes that happened whith the blood pressure due to pregnancy. Polemical aspects in the procedure of blood pressure measurement are discussed; for example, which one of the Korotkoff phases (4 or 5) that better represent the diastolic blood pressure and the use of Ambulatory Blood Pressure Monitoring in pregnancy. The recommendations from different societies are emphasized (American Heart Association, British Hypertension Society, Australasian Society, National High Blood Pressure Education Program and World Health Organization). 


\section{TOMA DE LA PRESIÓN ARTERIAL EN EMBARAZADAS}

Se trata de una revisión de la literatura con respeto a la toma indirecta de la presión arterial (PA) en embarazadas normotensas. Se describen los cámbios que ocurrer en la presión arterial sistólica y diastólica a causa del embarazo. Son discutidos aspectos polémicos en el procedimiento de la toma de la PA, como por ejemplo: cuál es la fase de los sonidos de Korotkoff (fases cuatro o cinco) que representa mejor la presión sistólica. Se enfatizan las recomendaciones de diferentes sociedades (American Heart Association, British Hypertension Society, Australasian Society, National High Blood Pressure Education Program and World Health Organization).

TÉRMINOS CLAVES: presión arterial, toma de presión en embarazadas

\section{REFERÊNCIAS BIBLIOGRÁFICAS}

01. AFTERY, E.B.; WARD, A.P. The indirect method of recording blood pressure. Cardiovasc.Res., v. 2, p. 210-8, 1968.

02. AMODEO, C. MAPA: histórico, indicações e limitações. In: MION Jr, D. et al. MAPA: monitorização ambulatorial da pressão arterial. São Paulo: Atheneu, 1995. cap. 6, p. 45-8.

03. ANDROS, G.J.; MICH, A.A. Blood pressure in normal pregnancy. Am.J.Obstet.Gynecol, v. 50, p.300-6, 1945.

04. AUSTRALASIAN SOCIETY FOR THE STUDY OF HYPERTENSION IN PREGNANCY. Management of hypertension in pregnancy: executive summary. Med.J.Aust., v. 158, n.10, p. 700-2, May 1993.

05. BARRON, W.M.; LINDHEIMER, M.D. Management of hypertension during pregnancy. In: LARAGH, J.H.; BRENNER, B.M. Hypertension: pathophysiology, diagnosis and management. 2. ed. New York: Raven, 1995. cap. 143, p.2427-50.

06. BEREZOWSKI, A. et al. Evolução da pressão arterial durante a gestação em grávidas normais. J.Bras.Ginecol., v.99, n.5, p. 187-90, 1989.

07. BISSON, D.L. et al. Conflicting views on the meassurement of blood pressure in pregnancy. Br.J.Obstet.Gynaecol., v.98, p. 842-3, 1991.

08. BRASIL. Ministério da Saúde. Secretaria da Assistência à Saúde. Departamento de Programas de Saúde. Coordenação de Doenças Cardiovasculares. Controle da hipertensão arterial: uma proposta de integração ensinoserviço. Rio de Janeiro, CDCV/NUTES/MS, 1993.

09. BROWN, M.A.; WHITWORTH, J.A. Recording diastolic blood pressure in pregnancy. Br.Med.J., v. 303, n. 6794, p. 120-1, July, 1991.

10. BROWN, M.A. et al. Ambulatory blood pressure monitoring during pregnancy: comparison with mercury sphygmomanometry. Am.J.Hypertens, n. 9, v. 6, p. 745-9, 1993.
11. BROWN, M. A. et al. Measuring blood pressure in pregnant women: a comparison of direct and indirect methods. Am.J.Obstet.Gynecol., v.171, n.3, p. 661-7, 1994.

12. CLARK,S. et al. Ambulatory blood pressure monitoring during pregnancy: validation of the TM - 2420 monitor. Obstet.Gynecol., v. 77, n. 1, p.152-5, 1991.

13. HALLIGAN, A. et al. Twenty four - hour ambulatory blood pressure measurement in a primigravid population. J.Hypertens., v. 11, n. 8, p. 869-73, 1993.

14. HARE, D.C.; KARN, M.N. An investigation of blood pressure, pulse rate, and the response to exercise during normal pregnancy, and some observations after confinement. Q.J.Med., v. 22, p. 381-404, 1929.

15. HENRY, J.S. The effect of pregnancy upon the blood pressure. Br.J.Obstet.Gynaecol., v. 43, p. 90824, 1936.

16. JOHENNING, A.R.; BARRON, W.M. Indirect blood pressure measurement in pregnancy: Korotkoff phase 4 versus phase 5. Am.J.Obstet.Gynecol., v. 167, n.3, p. 577-80, 1992.

17. LOPES, M.C. et al. The measurement of diastolic blood pressure during pregnancy: wich Korotkoff phase should be used. Am.J.Obstet.Gynecol., v.170, n. 2, p. 574-8, 1994.

18. LUDERS, C. et al. Mapa na gestante. In: MION Jr., D. et al. MAPA: monitorização ambulatorial da pressão arterial. São Paulo: Atheneu, 1995. cap. 12, p. 83-8.

19. MACGILLIVRAY, G. et al. Blood pressure survey in pregnacy. Clin.Sci., v. 37, p. 395-407, 1969.

20. MANCIA, G.; ZANCHETTI, A. Ambulatory blood pressure monitoring and hypertension. Clin.Exp.Hypertens.Theory Pract., A 7, p. 1467, 1985./ Apresentado no Satellite Symposium 10th Annual Meeting International Society of Hypertension, Stresa, Italy, 1984/.

21. MARGULIES, M. et al. Arterial blood pressure standards during normal pregnancy and their relation with mother - fetus variables. Am.J.Obstet.Gynecol., v. 156, n. 5, p. 1105-9, 1987. 
22. MARTINS, J.A.P. Manual de obstetrícia. São Paulo: EPU, 1982. v. 1. cap. 5, p. 99-127: Modificações do organismo materno.

23. MILSOM, I. et al. An evaluation of automated indirect blood pressure measurement during pregnacy. Acta Obstet.Gynecol.Scand., v. 65, n. 7, p. 7215, 1986.

24. NATIONAL high blood pressure education program. Part 1 Am.J.Obstet.Gynecol., v. 163, n. 5, p. 1691-712, 1990.

25. NEME, B. Obstetrícia básica. São Paulo: Sarvier, 1994. cap. 30, p. 216-43: Doença hipertensiva específica da gestação.

26. OKONOFUA, F.E. et al. Blood pressure changes during pregnancy in nigerian women. Int.J.Cardiol., v. 37, n. 3, p. 373-9, 1992.

27. OLOFSSON, P.; PERSSON, K. A comparison between conventional and 24-hour automatic blood pressure monitoring in hypertensive pregnancy. Acta Obstet.Gynecol.Scand., v. 74, n. 6, p. 429-33, 1995.

28. PERLOFF, D. et al. Human blood pressure determination by sphygmomanometry. Circulation., v. 88, n. 5, p. 2460-70, 1993.

29. PERRY, I.J. et al. Recording diastolic blood pressure in pregnancy. Br.Med.J., v. 301, n 6762, p. 1198, 1990.

30. PERRY, I.J. et al. Conflicting views on the measurement of blood pressure in pregnancy. Br.J.Obstet. Gynaecol., v. 98, n. 3, p.241-3, 1991a.

31. PERRY, I.J. et al. Recording diastolic blood pressure in pregnancy. Br.Med.J., v. 302, n. 6769, p. 17980, $1991 \mathrm{~b}$.

32. PETRIE, J.C. et al. Recomendations on blood pressure measurement. British Hypertension Society. Br. Med. J., v. 293, p. 611-5, 1986.

33. RAYBURN, W.F.; ZUSPAN, F.P. Portable blood pressure monitoring for boderline or mild hypertension during pregnancy. Clin.Obstet. Gynecol., v. 35, n. 2, p. 395-401, 1992.
34. REISS, R.E. et al. Retrospective comparison of blood pressure course during preeclamptic and matched control pregnancies. Am.J.Obstet.Gynecol., v. 156, n. 4, p. 894-8, April 1987.

35. RUDGE, M.V.C.; BEREZOWSKI, A.T. Adaptação do organismo materno à gravidez. In: NEME, B. Obstetrícia básica. São Paulo: Savier, 1994. cap. 6, p. 31-7.

36. VILLAR, J. et al. The measuring of blood pressure during pregnancy. Am.J.Obstet.Gynecol., v. 161, n. 4, p. 1019-24, 1989.

37. VOLDMAN, E.S. et al. Valores de presion arterial normal en 1000 embarazadas primigestas de la consulta prenatal. Rev.Obstet.Ginecol. Venezuela, v. 48, p. 1-7, 1988.

38. WALlENBURG, H.C.S. Prevention of hypertensive disordes in pregnancy. Clin.Exp.Hipertens.Pregnancy. B, v. 7, n. 1/ 2, p. 121, 1988.

39. WICHMAN, K.; RYDÉN, G. Blood pressure and renal function during normal pregnancy. Acta Obstet.Gynecol.Scand., v. 65, n. 6, p. 561-6, 1986.

40. WILSON, M. et al. Blood pressure, the renin aldosterone system and sex steroids throughout normal pregnancy. Am.J.Med., v. 68, p. 97104, 1980

41. WORLD HEALTH ORGANIZATION. The hypertensive disorders of pregnancy. Geneva, 1987.( Technical Report Series, n. 758).

42. WORLD HEALTH ORGANIZATION. Geographic variation in the incidence of hypertension. Am.J.Obstet.Gynecol., v. 158, n.1, p. 80-3, 1988.

43. ZANCHETTI, A.; MANCIA, G. The centenary of blood pressure measurement: a tribute to Scipione Riva-Rocci. J.Hypertens., v. 14, n.1, p.1-12, 1996. 